\title{
RESEARCH HIGHLIGHT How to dodge pyroptosis: lessons from Shigella flexneri
}

Filip Van Hauwermeiren ${ }^{1}$ and Mohamed Lamkanfi $\mathbb{I D}^{2 凶}$

(c) CEMCS, CAS 2021

Cell Research (2022) 32:227-228; https://doi.org/10.1038/s41422-021-00607-5

\begin{abstract}
Bacillary dysentery is an intestinal infection caused by Shigella, a group of Gram-negative bacteria that roam freely in the cytosol of infected host cells. A recent publication by Li et al. in Nature shows that Shigella flexneri actively targets caspase-4/11 via a novel posttranslational modification to dodge pyroptosis induction by the noncanonical inflammasome.
\end{abstract}

Inflammasomes are at the frontline of the body's protective response against tissue injury and pathogenic microorganisms. These large multiprotein complexes assemble in the cytosol of myeloid and epithelial cells and engage inflammatory caspases when signs of imminent threat are detected. Canonical inflammasomes mediate recruitment and activation of caspase-1. ${ }^{1,2}$ In another major inflammasome axis termed the 'noncanonical inflammasome pathway', members of the guanylate-binding protein (GBP) family coordinate the release and presentation of lipopolysaccharides (LPS) - a core component of the cell wall of Gram-negative bacteria - to caspase-4/11. ${ }^{3,4}$ Inflammasome activation has strong pro-inflammatory consequences because caspase- 1 matures the inflammatory cytokines interleukin (IL)-1 $\beta$ and IL-18. In addition, murine caspases 1 and 11 and their human orthologs caspases 1, 4 and 5 cleave gasdermin D (GSDMD), the liberated amino-terminal domain of which readily translocates to the plasma membrane and forms large oligomeric pores that induce pyroptosis (Fig. 1). GSDMD pores also allow for $\mathrm{K}^{+}$efflux to occur, consequentially activating the NLRP3 inflammasome. ${ }^{5}$ Pyroptosis frequently promotes protective antimicrobial defenses in the context of localized infections, but uncontrolled pyroptosis induction can also drive pathological tissue damage in chronic auto-inflammatory diseases and in the context of sepsis and severe, life-threatening infections. ${ }^{\prime}$

A recent study by $\mathrm{Li}$ et al. ${ }^{6}$ in Nature now describes how the Gram-negative pathogen Shigella flexneri, a causative agent for bacillary dysentery, dodges pyroptosis induction by the noncanonical inflammasome to roam freely and replicate in the cytosol of infected host cells. The authors start off with the unexpected observation that caspase-11-sufficient and -knockout mice are equally susceptible to intraperitoneal S. flexneri infection, whereas host lethality caused by infection with Burkholderia thailandensis - a Gram-negative bacterium that also invades the cytosol - is significantly higher in caspase-11-deficient mice. A simple explanation for these opposite outcomes might have been that S. flexneri LPS is incapable of inducing caspase-11 activation. However, transfection of purified S. flexneri LPS proved fully capable of inducing noncanonical inflammasome activation in the absence of infection. This suggests that a more sophisticated infection-propagating mechanism is at play through which $S$. flexneri actively interferes with LPS recognition or by which it suppresses downstream steps that would otherwise lead to activation of the noncanonical inflammasome. To better delineate the stealth mechanisms enabling S. flexneri to live in the host cytosol without inducing pyroptosis, the authors turned their attention to a full inspection of previously identified S. flexneri virulence factors. These efforts resulted in the identification of OspC3 as the protein that enables $S$. flexneri to suppress pyroptosis. This conclusion is grounded in the observation that engineered OspC3-deficient Shigella bacteria were no longer able to evade pyroptosis induction. Moreover, transgenic expression of OspC3 was sufficient to blunt induction of pyroptosis by cytosolic LPS delivery and in macrophages infected with the unrelated Gram-negative pathogen Salmonella Typhimurium.

Meticulously designed biochemical studies provided a detailed molecular picture of how OspC3 accomplishes the remarkable feat of halting pyroptosis induction by S. flexneri despite its LPS being recognized in the cytosol. Mass spectrometry measurements revealed that $\mathrm{OspC} 3$ catalyzes a novel type of posttranslational modification (PTM), which the authors coined arginine 'ADPriboxanation' and which they showed is used by $S$. flexneri to modify specific arginine residues in human caspase-4 (Arg314) and murine caspase-11 (Arg310). These modifications result in a dual inhibitory effect: (i) they block caspase-4/11 auto-cleavage and thus noncanonical inflammasome activation; ${ }^{7}$ and (ii) they disrupt docking of GSDMD into the caspase-4/11 exosite, ${ }^{8}$ thereby hampering GSDMD cleavage and pore assembly (Fig. 1).

Consistent with pyroptosis being rescued when OspC3 is inactivated, almost all mice infected with Shigella mutants expressing catalytically inactive OspC3 survived, whereas nearly all animals infected with wild-type Shigella succumbed to the infection. Moreover, caspase-11 and GSDMD activation drove significantly increased production of anti-Shigella antibodies in mice infected with the OspC3 mutant compared to those infected with wild-type Shigella. These findings suggest an important role for pyroptosis in boosting protective humoral immunity against $S$. flexneri, although additional work is needed to clarify the effector mechanisms by which GSDMD activation enhances antigenspecific antibody production in infected mice. Spurred by the potential clinical impact of their findings, the authors next demonstrated that a candidate vaccine strain in which OspC3 was mutated boosted the levels of Shigella-targeting antibodies in vaccinated mice and provided increased protection against rechallenge with wild-type $S$. flexneri. Considering that shigellosis remains an important cause of morbidity and mortality among

\footnotetext{
'Janssen Immunosciences, World Without Disease Accelerator, Pharmaceutical Companies of Johnson \& Johnson, Beerse, Belgium. ${ }^{2}$ Laboratory of Medical Immunology, Department of Internal Medicine and Paediatrics, Ghent University, Ghent, Belgium. ${ }^{凶}$ email: mohamed.lamkanfi@ugent.be
} 


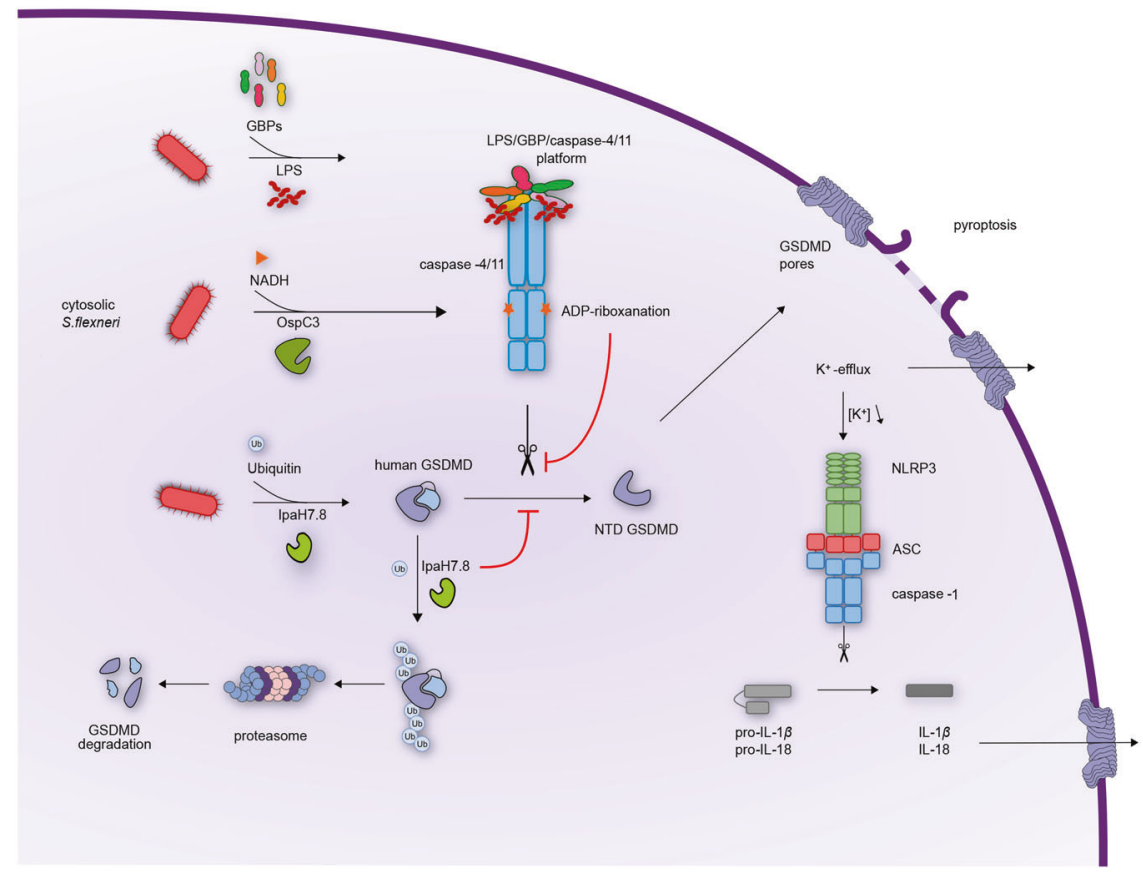

Fig. 1 Shigella virulence mechanisms to inhibit pyroptosis. LPS from S. flexneri is detected in the host cytosol, but the pathogen expresses two virulence factors that actively suppress caspase-4/11 activation and assembly of GSDMD pores, thereby inhibiting pyroptosis and secondary activation of the NLRP3 inflammasome. S. flexneri OspC3 is an enzyme that modifies Arg314 in human caspase-4 and Arg310 in murine caspase-11 by ADP-riboxanation. This posttranslational modification interferes with caspase-4/11 auto-cleavage and recruitment of GSDMD. The virulence factor S. flexneri IpaH7.8 hijacks the host ubiquitin-proteasome system to ubiquitylate and degrade GSDMD in human, but not rodent cells.

young children in developing countries, these important insights may greatly assist in the development of improved Shigella vaccine strains with enhanced ability to mount protective humoral and $T$ cell immunity and increased clinical efficacy.

Notably, Li and colleagues identified at least 27 OspC3 homologs in diverse bacterial species, a subset of which they showed can readily ADP-riboxanate caspase- 11 . This highlights ADP-riboxanation of caspase-4/11 as a widely spread virulence strategy to evade pyroptosis. Remarkably, another recent report showed that $S$. flexneri expresses an additional virulence factor named IpaH7.8 that ubiquitinates and targets human GSDMD for degradation by the host's proteasome system (Fig. 1). ${ }^{9}$ Mouse GSDMD, however, was not targeted by IpaH7.8, explaining why inactivation of OspC3 was sufficient to rescue pyroptosis induction in S. flexneri-infected murine macrophages. This differential sensitivity of human and rodent GSDMD to IpaH7.8-mediated degradation may also explain why humans are significantly more susceptible to shigellosis than rodents.

In conclusion, the recent reports by $\mathrm{Li}$ and colleagues ${ }^{6}$ and Luchetti and colleagues ${ }^{9}$ have uncovered two complementary virulence mechanisms through which $S$. flexneri evades pyroptosis in order to flourish and replicate in its hosts. These novel insights emphasize the importance of pyroptosis in anti-bacterial host defense, and may inspire novel therapeutic strategies to inhibit pyroptosis in chronic inflammatory diseases. Finally, these pioneering reports open up novel avenues for future research into how ADP-riboxanation and ubiquitination are exploited by microbial pathogens to manipulate and hijack additional host defense mechanisms to take the upper hand in the perpetuous war between hosts and pathogens.

\section{REFERENCES}

1. Van Opdenbosch, N. \& Lamkanfi, M. Immunity 50, 1352-1364 (2019).

2. Lamkanfi, M. \& Dixit, V. M. Cell 157, 1013-1022 (2014).

3. Shi, J. et al. Nature 514, 187-192 (2014).

4. Santos, J. C. et al. Nat. Commun. 11, 3276 (2020).

5. Kayagaki, N. et al. Nature 479, 117-121 (2011).

6. Li, Z. et al. Nature 599, 290-295 (2021).

7. Lee, B. L. et al. J. Exp. Med. 215, 2279-2288 (2018).

8. Vande Walle, L. \& Lamkanfi, M. Immunity 53, 6-8 (2020).

9. Luchetti, G. et al. Cell Host Microbe 29, 1521-1530.e1510 (2021).

\section{ACKNOWLEDGEMENTS}

Research in the Lamkanfi lab is supported by grants from the European Research Council (683144) and the Fund for Scientific Research-Flanders (G014221N and G017121N).

\section{ADDITIONAL INFORMATION}

Correspondence and requests for materials should be addressed to Mohamed Lamkanfi.

Reprints and permission information is available at http://www.nature.com/ reprints 